\title{
Classification, diagnosis and treatment of ACTH-independent macronodular adrenal hyperplasia
}

\author{
Heng-Chuan Su, MM; Jun Dai, MM; Xin Huang, MM; Wen-long Zhou, MM; Bao-xing Huang, MM; \\ Wan-li Cao, MM; Fu-Kang Sun, MD
}

Department of Urology, Ruiijn Hospital, School of Medicine, Shanghai Jiaotong University, Shanghai, China

Cite as: Can Urol Assoc J 2013;7(9-10):e594-7. http://dx.doi.org/10.5489/cuaj.420

Published online September 10, 2013.

\section{Abstract}

ACTH-independent macronodular adrenal hyperplasia (AIMAH) is a distinctive subtype of Cushing's syndrome (CS), with different clinical manifestations according to the level of serum cortisol. Based on clinical manifestations and serum cortisol, we divide AIMAH into three types, subclinical AIMAH, clinical AIMAH and high-risk AIMAH, with varied treatment methods being adapted to different subtypes. At the same time, we describe 3 patients who represent these subtypes of this disease, and review some cases of AIMAH which have been previously reported in the English literature. To our knowledge, this is the first article discussing classification, diagnosis and treatment of this disease and should be useful for future therapy of AIMAH.

\section{Introduction}

AIMAH is a rare form of Cushing's syndrome (CS). Its main clinical features are: (1) male dominant; (2) usually a higher mean age; (3) autonomous cortisol production accompanied by a low ACTH concentration and no suppression by dexamethasone; (4) no abnormality in the pituitary region; and (5) marked enlargement of the adrenal glands and increased isotope uptake on adrenal scintigraphy. ${ }^{1}$

However, in our clinical practice, we found many patients with AIMAH initially presenting with normal endrocine examinations. Several years later, some patients developed clinical AIMAH. The diagnosis and treatment of these patients were different from those of clinical AIMAH. Therefore, we divide AIMAH into 3 types: (1) subclinical AIMAH with non-specific syndromes, such as hypertension and diabetes; (2) clinical AIMAH with typical CS and abnormal endocrine examinations; and (3) high-risk AIMAH with typical CS and severe complications caused by high serum cortisol. Each subtype requires varied treatment methods. To elaborate our experience systematically, we detail the clinical symptoms and treatment of these cases.

\section{Case report}

\section{Case 1}

A 51-year-old man was referred to the hospital for bilateral adrenal masses which was found unintentionally during a routine physical examination. After admission, physical findings revealed high blood pressure. On laboratory examination, the patient showed increased fasting plasma glucose (11.92 $\mathrm{mmol} / \mathrm{L}$, normal range: 3.9-6.1 $\mathrm{mmol} / \mathrm{L})$, a partially decreased adrenocorticotropic hormone (ACTH) $(7.51 \mathrm{pg} / \mathrm{mL}$, normal range: $12.0-78.0 \mathrm{pg} / \mathrm{mL}$ ) and subnormal suppression following low-dose overnight dexamethasone suppression (1 mg:5.02 ug/dL, $2 \mathrm{mg}: 3.58 \mathrm{ug} / \mathrm{dL}$ ). The circadian rhythm in cortisol secretion was normal. The computed tomography (CT) scan revealed bilaterally enlarged adrenal glands with multiple ginger-like nodules. Excluding primary pigmented nodular adrenocortical disease, this patient was diagnosed with subclinical AIMAH and accepted antihypertensive and hypoglycemic treatment. Seventeen days later, he was discharged from hospital with normal blood pressure and blood glucose. During the 13-month follow-up, his blood pressure, blood glucose and endocrine examinations went back to normal and his CT scan showed no enlargement in the bilateral adrenal gland.

\section{Case 2}

A 48-year-old male was referred to our hospital for hypertension and edema in both legs. Physical findings revealed 
central obesity, thinning of the skin, buffalo hump and high blood pressure. On laboratory examination, the patient showed decreased ACTH $(2.45 \mathrm{pg} / \mathrm{mL})$, elevated levels of plasma cortisol (29.58 ug/dL, normal range: $6.7-22.6 \mathrm{ug} / \mathrm{dL}$ ) and 24-hour urinary free cortisol (450 umol, normal range: 21-111 umol). Normal circadian rhythm in cortisol secretion was lost (8 am:29.58 ug/dL, 4 pm:27.64 ug/dL, $0 \mathrm{am}: 26.89 \mathrm{ug} / \mathrm{dL}$ ). There was no suppression of cortisol secretion with low- and high-dose overnight dexamethasone suppression tests ( $2 \mathrm{mg}: 24.89 \mathrm{ug} / \mathrm{dL}, 8 \mathrm{mg}: 23.79 \mathrm{ug} / \mathrm{dL}$ ). The CT scan revealed bilaterally enlarged adrenal glands accompanied by multiple ginger-like nodules and no abnormalities in the pituitary gland. This patient was diagnosed with clinical AIMAH and accepted unilateral adrenalectomy. The resected adrenal gland weighed $35 \mathrm{~g}$ and contained multiple macroscopic nodules. Pathologically, the nodules consisted of clear-type cells and compact-type cells, compressing the surrounding cortex. At the 2-year follow-up, the patient recovered well, with normal endrocrine function and blood pressure His CT scan showed no enlargement in the contralateral adrenal gland.

\section{Case 3}

A 55-year-old female was referred to our hospital for hypertension and rapid weight gain, accompanied by cardiorespiratory dysfunction for 1 year, chronic hepatitis B for 3 years and chronic nephritis for 30 years. Physical findings revealed central obesity, multiple bruises, thinning of the skin, buffalo hump and high blood pressure (200/110 $\mathrm{mmHg})$. On laboratory examination, plasma cortisol was $39.58 \mathrm{ug} / \mathrm{dL}$ and 24-hour urinary free cortisol was 720 umol. Normal circadian rhythm in cortisol secretion was lost (8 am:39.58 ug/dL, 4 pm:37.64 ug/dL, 0 am: $36.89 u g / d L)$. There was no suppression of cortisol secretion with low- and high-dose overnight dexamethasone suppression test (2 mg:35.89 ug/dL, $8 \mathrm{mg}: 33.79 \mathrm{ug} / \mathrm{dL}$ ). The CT scan revealed bilaterally enlarged adrenal glands and no abnormalities in the pituitary gland. Cardiac ultrasound showed left ventricular hypertrophy and dilated left atrium. Based on these appearances, a diagnosis of high-risk AIMAH was made.

Initially, ketoconazole (800 mg/daily) was used to inhibit adrenal steroid production to improve the patient's general condition. A week later, serum cortisol decreased $(24.08 \mathrm{ug} / \mathrm{dL})$ paralleled by a deterioration of hepatic function. Consequently, the patient stopped ketoconazole and received supportive liver protection therapy. However, serum cortisol bounced back severely (54.66 ug/dL) accompanied by fluid retention, heart and lung failure within 20 days. She was administered brain natriuretic peptide (200 ug, $9 \mathrm{~mL} / \mathrm{h}$ with syringe pump) to improve the cardiorespiratory function, followed by unilateral adrenalectomy to decrease high cortisol level. The resected gland was $10 \times 10 \mathrm{~cm}$ in size, weighing $67.5 \mathrm{~g}$ and consisted of multiple nodules. Histological examination revealed that the nodules comprised mostly adrenocortical clear-type cells and some areas of compact-type cells.

The patient continued to take ketoconazole when her liver function was normalized. This patient was discharged at postoperative day 50. During the 10-month follow-up, the patient recovered well, with plasma cortisol at 10.75$26.79 \mathrm{ug} / \mathrm{dL}, 24$-hour urinary free cortisol 30.88-105.65 ug, blood pressure 100-160/70-100 mmHg. The CT scan showed no enlargement in the contralateral adrenal gland.

\section{Discussion}

At present, there is no standard to differentiate subclinical AIMAH, clinical AIMAH and high-risk AIMAH. Lacroix and colleagues reported that subclinical AIMAH patients usually present with slightly elevated midnight plasma or salivary cortisol, subnormal suppression following $1 \mathrm{mg}$ overnight dexamethasone suppression test, a partially suppressed ACTH, normal 24-hour urinary free cortisol production and absence of clinical signs of CS, paralleled by non-specific syndromes, such as hypertension and diabetes. ${ }^{2}$ Clinical AIMAH patients present with both CS and abnormal endocrine examinations. High-risk AIMAH patients have severe syndromes caused by high serum cortisol, such as hypokalemia, severe hypertension, clinical CS, and exhaustion of multiple organs, paralleled by obvious abnormity in endocrine examinations.

Pathologically, these three kinds were similar. The combined adrenal weight is usually $>60 \mathrm{~g}$ and can reach $200 \mathrm{~g}$ per gland (Fig. 1). ${ }^{3}$ On cut sections, the nodules are yellow due to their high lipid content. The nodules are composed of two cell types: either with clear cytoplasm (lipid-rich) that form cordon nest-like structures (Fig. 2, part A), or with compact cytoplasm (lipid-poor) that form nest- or island-like structures (Fig. 2, part B). The radiological patterns of these three kinds were also similar, showing bilaterally enlarged adrenal glands with multiple nodules like ginger (Fig. 3).

\section{Subclinical AIMAH}

In patients with subclinical AIMAH, the decision to treat should be bases on the age of the patient and morbidity associated with the hypercortisolic state (hypertension, diabetes, and neuropsychological manifestations). AIMAH progresses slowly. Ohashi and colleagues reported one subclinical AIMAH patient who developed into overt CS over 7 years. ${ }^{4}$ In our opinion, symptomatic treatment is the best choice for subclinical AIMAH. Sasao and colleagues and Lee and colleagues respectively reported one subclinical AIMAH patient who initially received symptomatic treatment for 7 years and 2 years, respectively. ${ }^{5,6}$ When the disease progressed 


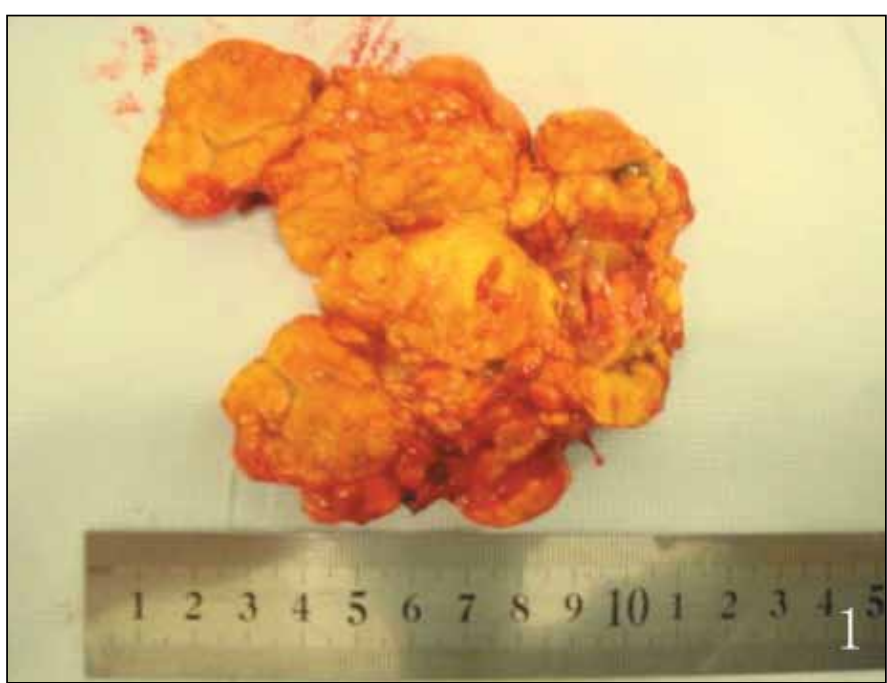

Fig. 1. The gross appearance of resected adrenal showed typical macronodular adrenal hyperplasia.

into overt CS, these 2 patients accepted surgery. Following the surgery, they recovered quickly. Close follow-up with semiannual adrenal CT scans and biochemical assessment are required in these patients and surgery is recommended when CS appears.

\section{Clinical AIMAH}

Standard therapy for clinical AIMAH is bilateral adrenalectomy, which can remove the source of serum cortisol radically, with 5 -year survival rates being $66 \%$ to $70 \%$. Ogura and colleagues have reported unilateral or subtotal adrenalectomy as another choice of therapy, which could avoid life-long glucocorticoid replacement and is effective in improving abnormal cortisol secretion. ${ }^{7}$ In our opinion, it is reasonable to remove the obviously diseased adrenal gland and first confirm it pathologically. Whether to remove the other adrenal gland depends on clinical and biochemical changes at the later time. When serum cortisol or blood pressure of this patient is still hard to control, we would remove the other adrenal gland. It is better to handle the adrenal gland at the right and then left when there are no differences between the two sides. This is due to the liver and vena cava being located on the right side of human body, making it difficult to operate again if the disease reoccurs.

\section{High-risk AIMAH}

Ameliorating high serum cortisol, improving organ function with medicine, and selecting an appropriate time to perform the operation are keys to cure high-risk AIMAH. Once AIMAH develops into high-risk AIMAH, the disease would develop quickly, accompanied by hypokalemia, hyperglycemia, severe hypertension and exhaustion of multiple

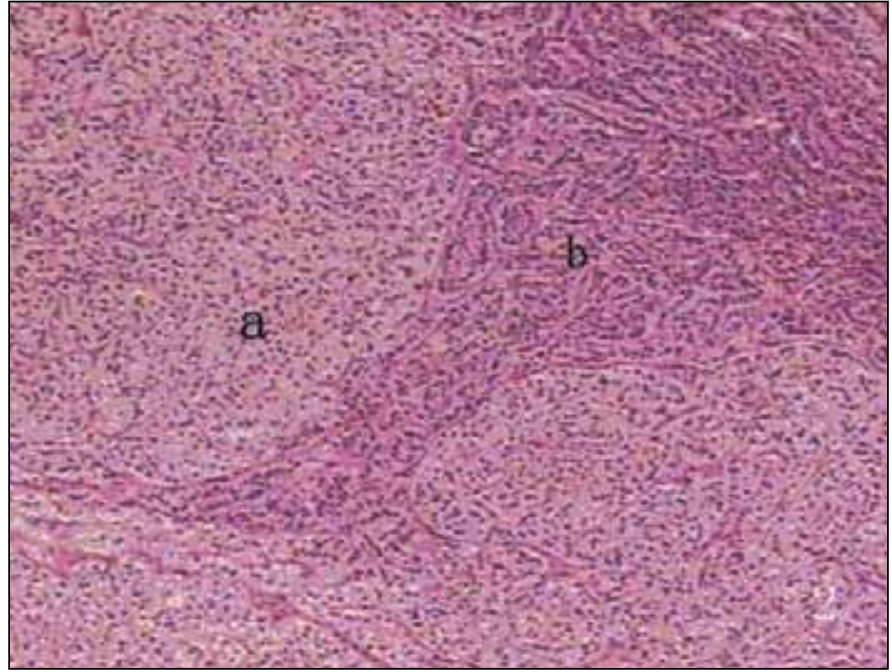

Fig. 2. The nodules are composed of two cell types: either with clear cytoplasm (lipid-rich) that form cordon nest-like structures (a), or with compact cytoplasm (lipid-poor) that form nest-or island-like structures (b).

organs. Case 3 is a high-risk AIMAH patient. Her disease aggravated progressively within 6 months, accompanied by rapid weight gain, abdominal striae and high blood pressure which was difficult to control. Plasma cortisol increased from $24.08 \mathrm{ug} / \mathrm{dL}$ to $54.66 \mathrm{ug} / \mathrm{dL}$ and her blood pressure increased to $230 / 170 \mathrm{mmHg}$, resulting in heart and respiratory failure within 20 days. The target organ should be resected as soon as possible once the cardio-pulmonary function improves, blood glucose levels are controlled at $7 \mathrm{mmol} / \mathrm{L}$, blood pressure is between 140-150/90-100mmHg, serum cortisol at 20-35 ud/dL and blood potassium is normal. The unilateral adrenalectomy is an effective treatment for high-risk AIMAH.

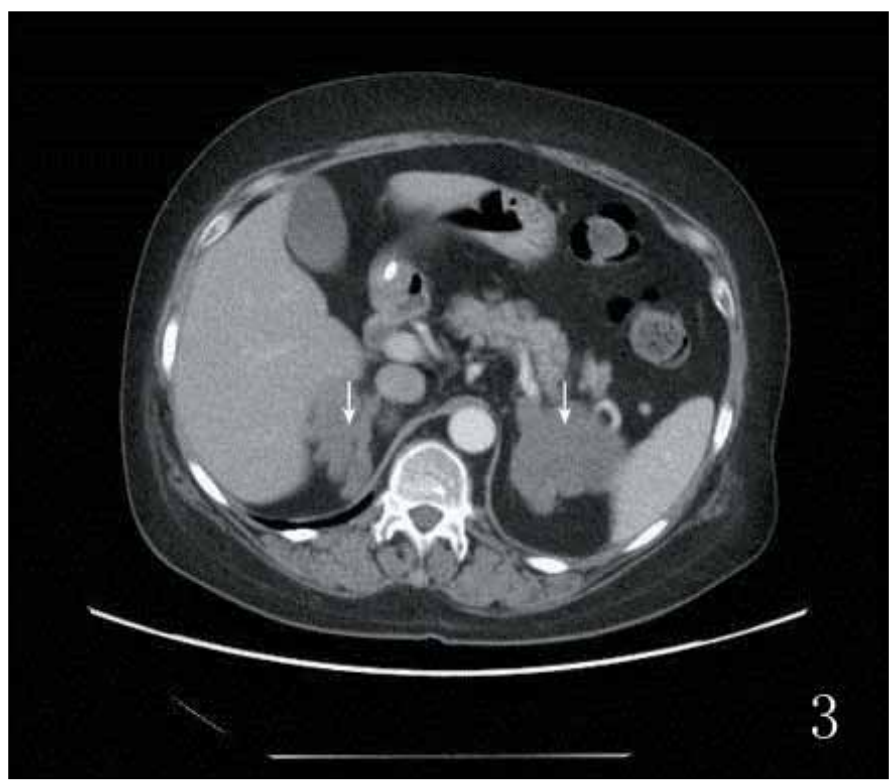

Fig. 3. The radiological patterns of these three subtypeswere similar, showing bilaterally enlarged adrenal glands with multiple nodules like ginger. 
There are various medications, such as ketoconazole, mitotane and metyrapone, to decrease serum cortisol for high-risk AIMAH. In case 3, we chose ketoconazole, which inhibits adrenal steroid production via the inhibition of multiple steroidogenic enzymes at the dose of $400 \mathrm{mg} /$ daily or $800 \mathrm{mg} /$ daily. ${ }^{8} \mathrm{~A}$ major side effect of ketoconazole is hepatotoxicity; therefore, liver function should be monitored routinely during treatment. Once hepatic and renal functions deteriorate, stop ketoconazole and start supportive liver protection.

\section{Therapy}

The identification of aberrant adrenal hormone receptors provides new opportunities for specific pharmacological therapies in AIMAH. ${ }^{2}$ Since multiple receptors could mediate cortisol hypersecretion in AIMAH, these patients should undergo screening for aberrant receptors, as this could affect the therapeutic strategy. Pharmaceutical intervention of the aberrantly expressed adrenal receptors in AIMAH has been attempted in only a few cases with conflicting results. ${ }^{9}$ Some patients responded successfully to this therapy and cortisol excess was controlled long-term, while in some patients the benefit lasted only a few months..$^{9,10}$

\section{Conclusion}

We do not have a lot of experience with AIMAH therapy; however, novel therapeutic agents remain to be tested, with the aim to cure patients in the future.

Competing interests: None declared.

This paper has been peer-reviewed.
Acknowledgements: This work was supported by the grant from the Shanghai Municipal Natural Science Foundation (No. 10411960000) and Shanghai Municipal Charitable Cancer Foundation. Dr. Heng-Chuan Su and Dr. Jun Dai contributed equally to this work.

\section{References}

1. Sato M, Soma M, Nakayama T, et al. A case of Adrenocorticotropin-independent Bilateral Adrenal Macronodular Hyperplasia (AIMAH) with Primary Hyperparathyroidism (PHPT). Endocr J 2006;53:1117. http://dx.doi.org/10.1507/endocri.53.111

2. Lacroix A. ACTH-independent macronodular adrenal hyperplasia. Best Pract Res Clin Endocrinol Metab 2009;23:245-59. http://dx.doi.org/10.1016/i.beem.2008.10.011

3. Malchoff CD, Malchoff DM. Adrenocorticotropic hormone-independent adrenal hyperplasia. Endocrinologist 1996;6:79-85. http://dx.doi.org/10.1097/00019616-199603000-00003

4. Ohashi A, Yamada Y, Sakaguchi K, et al. A natural history of adrenocorticotropin independent bilateral adrenal macronodular hyperplasia from preclinical to clinically overt Cushing's syndrome. Endocr I 2001;48:677-83. http://dx.doi.org/10.1507/endocri.48.677

5. Sasao T, Itoh N, Sato Y, et al. Subclinical Cushing syndrome due to adrenocorticotropic hormone-independent macronodular adrenocortical hyperplasia: changes in plasma cortisol levels during long-term follow-up. Urology 2000;55:145. http://dx.doi.org/10.1016/S0090-4295(99)00381-7

6. Lee KT, Arnott RD, McLean CA, et al. Corticotropin-independent macronodular adrenal hyperplasia associated with insulinoma. Endocr Pract 2011;17:e43-7. http://dx.doi.org/10.4158/EP10213.CR

7. Ogura $M$, Kusaka I, Nagasaka $S$, et al. Unilateral adrenalectomy improves insulin resistance and diabetes mellitus in a patient with ACTH-independent macronodular adrenal hyperplasia. Endocr J 2003;50:715-21. http://dx.doi.org/10.1507/endocri.50.715

8. Bertagna X, Guignat L, Groussin L, et al. Cushing's disease. Best Pract Res Clin Endocrinol Metab 2009;23:607-23. http://dx.doi.org/10.1016/i.beem.2009.06.001

9. Karapanou 0, Vlassopoulou B, Tzanela M, et al. ACTH-Independent Macronodular Adrenal Hyperplasia Due to Aberrant Receptor Expression: Is Medical Treatment Always an Option? Endocr Pract 2013;20:120.

10. Daidoh H, Morita H, Hanafusa J, et al. In vivo and in vitro effects of AVP and Vla receptor antagonist on Cushing's syndrome due to ACTH-independent macronodular adrenocortical hyperplasia. Clin Endocrinol (Oxf) 1998:48:403-9. http://dx.doi.org/10.1046/j.1365-2265.1998.00490.x

Correspondence: Dr. Fu-Kang Sun, Department of Urology, Ruijin Hospital, School of Medicine, Shanghai Jiaotong University, No. 197, Ruijin Er Road, Shanghai 200025, China; sunfukangb@126.com 